\title{
Características físico-químicas de méis produzidos em favos de diferentes idades
}

\section{Characteristics physicist-chemistries of honeys produced in combs different of the age}

\author{
Hélio Carlos Rocha ${ }^{1 *}$; Angélica de Almeida Lara ${ }^{2}$; \\ Dileta Cecchetti ${ }^{3}$; Alisson Becker Pacheco ${ }^{4}$
}

\begin{abstract}
Resumo
A cor do mel é uma característica relacionada à fonte floral e que mais interfere na preferência de compra do consumidor. Ao ser elaborado, o mel é depositado em favos de cera, a qual é composta de hidrocarbonetos, ésteres e uma pequena percentagem de ácidos graxos e alcoóis, que propiciam a cera a plasticidade e a capacidade de absorver diversos elementos. A reutilização consecutiva de favos pode transferir pigmentos ao mel, alterando as características do produto final. O presente estudo teve por objetivo avaliar a influência da reutilização de favos da melgueira sobre a coloração e as variáveis físico-químicas do mel. $\mathrm{O}$ experimento foi conduzido utilizando-se um delineamento em blocos casualizados com quatro tratamentos e seis repetições. Cada colméia foi considerada como um bloco. Os tratamentos constaram de caixilhos com lâminas de cera alveolada e favos com um, dois e três anos de uso, totalizando 24 caixilhos. Os méis foram avaliados através de espectrofotometria de reflectância difusa e as variáveis físico-químicas em laboratório por meio de procedimentos padrões. A reflectância difusa assim como o açúcar total, $\mathrm{pH}$, acidez e cinzas não apresentaram diferenças entre os tratamentos $(\mathrm{p}>0.05)$. A coloração do mel produzido em favos de diferentes anos de uso não sofreu alteração, porém o mel produzido em favos com lâminas de cera apresentou cor mais clara e menor conteúdo de açúcar total.
\end{abstract}

Palavras-chave: Coloração, cera, Apis mellifera L.

\begin{abstract}
Honey color is a characteristic related to the flower source and plays a major role on consumers buying preference. When finished, honey is placed in wax honeycombs that are composed mainly by hydrocarbons, esters and a small percentage of fatty acids and alcohols that give to wax the plasticity and ability to absorb several kinds of elements. The consecutive comb reutilization might transfer pigments to honey that, in turn, can alter honey final characteristics. In the present study the objective was to evaluate the influence of comb reutilization on honey physico-chemical and color characteristics. The experiment was carried out using randomized blocks with four treatments and six repetitions. Each hive was considered a block. The treatments consisted of boxes with sheet of alveolated wax and honeycomb with one, two or three years of usage, totaling 24 boxes. Honey samples were analyzed by diffuse reflectance spectrophotometry and the physico-chemical properties were analyzed by laboratory standard tests. The diffuse reflectance, total sugar, $\mathrm{pH}$, acidity and ash did not varied significantly among
\end{abstract}

\footnotetext{
${ }^{1}$ Dr. Professor dos Cursos de Agronomia e Medicina Veterinária da Universidade de Passo Fundo - UPF, Passo Fundo, RS, Brasil. Campus I, Bairro São José, BR 285, km 171. CEP 99001-970. Passo Fundo, RS. E-mail: helio@upf.br

${ }^{2}$ Eng.-Agr., Assistente Técnico do Projeto Apicultura - Universidade de Passo Fundo - FAMV/UPF. E-mail: lara@upf.br

${ }^{3}$ Msc. Professora do Instituto Federal do Rio Grande do Sul - E-mail: dileta.cecchetti@sertao.ifrs.edu.br

${ }^{4}$ Acadêmico do Curso de Agronomia - Universidade de Passo Fundo - FAMV/UPF. E-mail: alissonpacheco@ibest.com

* Autor para correspondência
} 
treatments $(\mathrm{p}>0.05)$. Honey color produced in honeycomb with different years of use was similar amongst treatment; however, the honey produced in sheets of alveolated wax had clearer color and less sugar content.

Key words: Color, wax, Apis mellifera $\mathrm{L}$.

O mel é uma das substâncias mais notáveis disponibilizado pela natureza ao homem e apreciado por milhões de pessoas, pode ser consumido hoje da mesma forma em que foi descoberto há milhares de anos atrás (CRANE, 1983; WHITE JUNIOR, 1992).

$\mathrm{O}$ aspecto de maior importância sensorial na aceitabilidade do mel é a coloração (ANACLETO; MARCHINI, 2004), seguida pelo estado de apresentação, com preferência para o produto não cristalizado. Pela percepção do olho humano, o mel no estado líquido é mais escuro do que na forma cristalizada, em função de que, àquele absorve e este reflete mais luz (ANACLETO; MARCHINI, 2004).

A coloração do mel pode variar em função da origem floral, desde o branco originário de flores de assa-peixe, ao âmbar de flores de laranjeira, ao escuro de flores silvestres e de eucalipto, ao preto de flores de trigo sarraceno (COUTO; COUTO, 1996).

$\mathrm{O}$ mel escuro apresenta maior quantidade de pigmentos vegetais, principalmente flavonóides e carotenóides, característicos antioxidantes naturais (CORBELLA; TEJERA; CERNUSCHI, 2005). Em temperaturas acima de $15^{\circ} \mathrm{C}$ é comum a mudança de estado, de coloração e de conteúdo no mel armazenado, no entanto, a principal contribuição na coloração do mel é devido a fonte floral (WHITE JUNIOR, 1992; COUTO; COUTO, 1996).

Um fator pouco estudado e que pode contribuir com a coloração do mel está relacionado com o manejo da cera nos caixilhos da melgueira. Muitos apicultores utilizam por vários anos os mesmos favos para a produção de mel em função da sua maior resistência durante o processo de centrifugação. Com o passar do tempo e uso a cera escurece e perde o aspecto de favo novo. A cera consiste em hidorcarbonetos, ésteres e uma pequena percentagem de ácidos graxos e alcoóis, sendo estes componentes secundários os que propiciam a plasticidade ao produto (BERRY; DELAPLANE, 2001) e a capacidade de absorver muitos tipos de materiais. Entre os materiais absorvidos estão esporos de fungos, bactérias, produtos químicos como os utilizados na produção vegetal e animal e metais pesados (BERRY; DELAPLANE, 2001). Em função destes aspectos, é possível que muitos pigmentos possam ser transferidos da cera para o mel, contribuindo desta forma para escurecer o produto. No presente trabalho estudou-se a influência da idade do favo da melgueira sobre a coloração e as variáveis físico-químicas do mel, contribuindo com conhecimentos que visem melhorar os procedimentos tecnológicos na exploração apícola.

O experimento foi conduzido na cidade de Passo Fundo, no estado do Rio Grande do Sul, utilizado-se seis colméias do modelo Langstroth. Os tratamentos constaram de lâminas de cera, e favos de melgueira com um, dois e três anos de uso, totalizando 24 caixilhos. Para evitar o efeito de fluxo de néctar de diferentes plantas, os tratamentos permaneceram no campo por um período de 32 dias, sendo a floração predominante de trevo branco (Tripholium repens L.) e eucalipto (Eucalyptus spp), carqueja (Baccharis trimera (Less.) D.C.), vassoura (Baccharis dracuncifolia D.C.) e mariamole (Senecio brasiliensis Less.).

$\mathrm{O}$ beneficiamento dos favos foi realizado em agroindústria com inspeção municipal e as análises físico-químicas em laboratório da FAMV/UPF. Os favos foram desoperculados e centrifugados individualmente sendo o mel acondicionado em vidros previamente identificados. A centrífuga após a extração do mel de cada tratamento foi 
lavada e limpa. Todas as amostras dos méis foram armazenadas em ambiente seco, ao abrigo da luz direta a $20^{\circ} \mathrm{C}$ até a sua análise que ocorreu três meses após a colheita.

A coloração do mel foi analisada por espectrofotometria de reflectância difusa com sensor ótico geométrico de esfera (modelo ColorQuest II Sphere (HUNTER LAB. EUA). As amostras foram transferidas para uma cubeta de quartzo de $50 \mathrm{~mm}$ e estas colocadas sobre o sensor ótico de 2,54 mm, realizando em cada amostra quatro leituras em duas posições diferentes de cada lado da cubeta. Quanto mais escuro for o mel menor é o valor da reflatância difusa e vice-versa.

As determinações de $\mathrm{pH}$, acidez, cinzas e açúcares seguiram os procedimentos padrões para análise de mel (BRASIL, 2000).

O delineamento experimental utilizado foi em blocos casualizados, com quatro tratamentos em seis repetições, sendo os dados submetidos a análise de variância e as médias comparadas através do teste de Tukey com nível de significância de 5\%.

Com o objetivo de medir a associação entre as variáveis: L* (luminosidade), açúcar total, $\mathrm{pH}$, acidez e cinzas foi calculado o coeficiente de correlação de Pearson, o qual, posteriormente, foi testado quanto a sua significância pelo teste $T$ (CARDELINO; ROVIRA, 1987).

O fluxo de néctar no decorrer do período experimental possibilitou às abelhas produzirem mel em quantidade suficiente para ocupar os favos da melgueira em curto espaço de tempo. Este período curto propiciou a contribuição de néctar de poucas espécies vegetais, como no presente trabalho.

As amostras de méis analisadas apresentaram o valor de 23,22 $\pm 0,43$ para luminosidade $\left(L^{*}\right)$, de $3,10 \pm 0,76$ para intensidade da cor vermelha $\left(\mathrm{a}^{*}\right) \mathrm{e}$ de 2,65 $\pm 0,64$ para intensidade da cor amarela $\left(b^{*}\right)$ (Tabela 1).

Tabela 1. Reflectância difusa, açúcar total, $\mathrm{pH}$, acidez e cinzas em méis produzidos em favos de melgueira de diferentes idades, Passo Fundo, RS

\begin{tabular}{lccccccc}
\hline Tratamentos & $\mathrm{L}^{*}$ & $\mathrm{a}^{*}$ & $\mathrm{~b}^{*}$ & $\begin{array}{c}\text { Açúcar total } \\
(\%)\end{array}$ & $\mathrm{pH}$ & $\begin{array}{c}\text { Acidez } \\
\left(\mathrm{meq}^{-1} \mathrm{~kg}^{-1}\right)\end{array}$ & $\begin{array}{c}\text { Cinzas } \\
(\%)\end{array}$ \\
\hline Lâmina de cera & $24,32 \pm 2,03$ & $2,96 \pm 0,63$ & $2,64 \pm 0,52$ & $77,6 \pm 0,78$ & $4,46 \pm 0,11$ & $42,7 \pm 6,71$ & $0,42 \pm 0,09$ \\
Favo 1 ano de uso & $23,24 \pm 0,35$ & $3,43 \pm 1,17$ & $2,84 \pm 1,01$ & $78,1 \pm 0,57$ & $4,44 \pm 0,14$ & $44,4 \pm 7,69$ & $0,43 \pm 0,11$ \\
Favo 2 anos de uso & $23,16 \pm 0,27$ & $2,99 \pm 0,54$ & $2,53 \pm 0,42$ & $78,0 \pm 0,68$ & $4,42 \pm 0,14$ & $48,6 \pm 8,43$ & $0,40 \pm 0,11$ \\
Favo 3 anos de uso & $23,71 \pm 0,42$ & $3,01 \pm 0,65$ & $2,59 \pm 0,59$ & $77,1 \pm 1,15$ & $4,41 \pm 0,13$ & $50,0 \pm 5,27$ & $0,41 \pm 0,06$ \\
Coeficiente & 3,21 & 0,76 & 0,64 & 3,44 & 0,56 & 16,08 & 15,54 \\
variação (\%) & 0,1628 & 0,4241 & 0,5651 & 0,1435 & 0,9407 & 0,3194 & 0,8685 \\
$p$-value & & & & & & \\
\hline
\end{tabular}

$\mathrm{L}^{*}$ (luminosidade), $\mathrm{a}^{*}$ (intensidade da cor vermelha) e $\mathrm{b}^{*}$ (intensidade da cor amarela). ${ }^{1}$ probabilidade de que a hipótese nula seja verdadeira.

As amostras de méis apresentaram 77,74 $\pm 0,88$ para açúcar total, de 4,44 $\pm 0,13$ para $\mathrm{pH}$, de 46,46 $\pm 7,30$ para acidez e de $0,42 \pm 0,09$ para cinzas (Tabela 1).

O açúcar total em 24 amostras de méis analisado apresentou uma amplitude de 75 a $79 \%$ dentro do padrão estabelecido pela norma vigente que estabelece o mínimo de 65\% (BRASIL, 2000). Valores semelhantes foram relatados por Marchini et al. (2001), com valores médios de 72,6 e 72,3 para mel de origem de flores silvestres e de eucalipto no estado de São Paulo.

$\mathrm{O}$ pH das 24 amostras de méis analisadas variaram de 4,26 a 4,65, estando dentro da norma 
que estabelece valores de 3,3 a 4,6 (BRASIL, 2000). Todavia, estes dados diferem de Marchini et al., (2001), que relataram valores médios de 3,2 e 3,6 obtido em mel de origem silvestre e de eucalipto no estado de São Paulo.

A acidez do mel foi de 33,4 a 58,7 0 meq. $\mathrm{kg}^{-1}$, revelando que $37,5 \%$ das amostras analisadas apresentam-se fora do padrão estabelecido pela norma vigente, que é no máximo de 50 meq. $\mathrm{kg}^{-1}$ (BRASIL, 2000). Cabe destacar que a lâmina de cera apresentou uma amostra acima do padrão, enquanto que favos com um ano, três e quatro anos, apresentaram respectivamente duas amostras e três amostras acima do limite estabelecido. Marchini et al. (2001), encontraram valores de 6,0 a 46,0 meq. $\mathrm{kg}^{-1}$ para amostras de méis do cerrado paulista.

É interessante destacar que há um incremento na acidez do mel na medida em que aumenta a idade dos favos da melgueira (Tabela 1), embora a correlação entre as variáveis L*(luminosidade) e acidez seja de 0,25 (P=0,224), não identificando uma associação.

Os valores para cinzas nos méis analisados apresentaram variaram de 0,29 a $0,59 \%$ e se encontra dentro do limite estabelecido de $0,6 \%$ para mel de origem floral (BRASIL, 2000). Marchini et al. (2001), trabalhando com méis do cerrado paulista, relatou que $8,8 \%$ das 34 amostras encontravam fora do padrão da norma vigente para o teor de cinzas.

Apesar de não haver diferença estatística entre o uso de lâmina de cera, favos com um, dois e três anos de uso, a lâmina de cera permitiu a produção de mel claro se comparado aos demais. Os méis claros apresentaram menor teor de açúcar total, com um coeficiente de correlação entre L* (luminosidade) e açúcar total de $-0,43(\mathrm{P}=0,033)$ (Tabela 2).

Tabela 2. Coeficiente de correlação de Pearson entre as variáveis L* (luminosidade), açúcar total, pH, acidez e cinzas de méis produzidos em favos de melgueira de diferentes idades.

\begin{tabular}{lccc}
\hline Contrastes & $\mathrm{n}^{\text {o }}$ de observações & $\mathrm{r}$ & -value $^{1}$ \\
\hline $\mathrm{L}^{*} \mathrm{x}$ açúcar total & 24 & $-0,43$ & 0,033 \\
$\mathrm{~L}^{*} \mathrm{x}$ pH & 24 & $-0,01$ & 0,978 \\
$\mathrm{~L}^{*} \mathrm{x}$ acidez & 24 & 0,25 & 0,224 \\
$\mathrm{~L}^{*}$ x cinzas & 24 & $-0,02$ & 0,933 \\
açúcar total x pH & 24 & 0,15 & 0,468 \\
açúcar total x acidez & 24 & 0,02 & 0,926 \\
açúcar total x cinzas & 24 & $-0,28$ & 0,183 \\
pH x acidez & 24 & 0,11 & 0,616 \\
pH x cinzas & 24 & 0,07 & 0,737 \\
acidez x cinzas & 24 & $-0,22$ & 0,286 \\
\hline
\end{tabular}

$r=$ coeficiente de correlação de Pearson.

${ }^{1}$ probabilidade de que a hipótese nula seja verdadeira.

González-Miret et al. (2005), em estudo sobre a relação da coloração de méis e conteúdo de minerais encontraram que a luminosidade $\left(\mathrm{L}^{*}\right)$ teve correlação com $\mathrm{S}, \mathrm{Ca}, \mathrm{Fe}, \mathrm{As}, \mathrm{Pb}$ e Cd para os méis do tipo escuro. Já os méis do tipo claros e âmbar, apresentaram uma baixa correlação de $\mathrm{C}_{\mathrm{ab}}{ }^{*}$ e $\mathrm{b}^{*}$ com o conteúdo de minerais.
A coloração do mel não sofreu alterações quando produzido em favos de melgueira de diferentes idades. No entanto, os méis claros foram produzidos em lâminas de cera, e apresentaram menor conteúdo de açúcar total do que os escuros. 


\section{Agradecimentos}

Os autores agradecem à Secretaria de Ciência e Tecnologia do RS, através do Programa Pólos de Inovação Tecnológica pelo apoio ao Projeto de Desenvolvimento da Apicultura na região do Planalto Médio do RS.

\section{Referências}

ANACLETO, D. A.; MARCHINI, L. C. Composição físico-química de amostras de méis de Apis mellifera $\mathrm{L}$. provenientes do cerrado paulista. Boletim da Indústria Animal, Piracicaba, v. 61, n. 2, p. 161-172, 2004.

BERRY, J.; DELAPLANE, K. S. Effects of comb age on honey bee colony growth and brood survivorship. Jornal of Apicultural Research, v. 40, n. 1, p. 3-7, 2001. Disponível em: http://www.ent.uga.edu/bees/ publications/effects_comb_age.pdf. Acesso em: 15 jan. 2009.

BRASIL. Ministério da Agricultura. Instrução Normativa $\mathrm{n}^{\mathrm{o}} 11$, de 20 de outubro de 2000. Regulamento técnico de identidade e qualidade do mel. 2000. Disponível em: http://extranet.agricultura.gov.br/sislegis-consulta/ consultarLegislacao. do operacao $=$ visualizar\&id $=7797$. Acesso em: 10 jan. de 2008.
CARDELINO, R.; ROVIRA, J. Mejoramiento genetico animal. Montevideo: Hemisfério Sur, 1987. 253 p.

CORBELLA, E.; TEJERA, L.; CERNUSCHI, F. Calidad y origem botánico de mieles del nordeste de Uruguay. Revista INIA, n. 3, p. 6-7, 2005. Disponível em: http:// www.inia.org.uy. Acesso em: 03 nov. 2005.

COUTO, R. H. N.; COUTO, L. A. Apicultura: manejo e produtos. Jaboticabal: FUNDEP, 1996. 154 p.

CRANE, E. O livro do mel. São Paulo: Livraria Nobel, 1983. $226 \mathrm{p}$.

GONZÁLEZ-MIRET, M. L.;TERRAB, A., HERNANZ, D.; MARIA Ä̈ NGELES FERNÄ̈ NDEZRECAMALES, M. A.; HEREDIA, F. J. Multivariate correlation betwen color and mineral composition of honeys and by their botanical origin. Journal of Agricultural and Food Chemistry, Sevilla, v. 53, n. 7, p. 2574-2580, 2005.

MARCHINI, L. C.; MORETI, A. C. C. C.; TEIXEIRA, E. W.; SILVA, E. C. A.; RODRIGUES, R. R.; SOUZA, V. C. Plantas visitadas por abelhas africanizadas em duas localidades do estado de São Paulo. Scientia Agrícola, Piracicaba, v. 58, n. 2, p. 413-420, 2001.

WHITE JÚNIOR, J. W. Honey. In: GRAHAM, J. M. The hive and the honey bee. Hamilton: Illinois, 1992, p. 869925. 
